\title{
Tangence
}

\section{Lire Claude Simon lisant Proust Reading Claude Simon reading Proust}

\section{Christine Genin}

Numéro 112, 2016

Les trajets de la lecture. Autour de Claude Simon

URI : https://id.erudit.org/iderudit/1039909ar

DOI : https://doi.org/10.7202/1039909ar

Aller au sommaire du numéro

\section{Éditeur(s)}

Tangence

ISSN

1189-4563 (imprimé)

1710-0305 (numérique)

Découvrir la revue

Citer cet article

Genin, C. (2016). Lire Claude Simon lisant Proust. Tangence, (112), 109-131. https://doi.org/10.7202/1039909ar

\section{Résumé de l'article}

Comme Roland Barthes, Claude Simon aurait pu écrire " Proust, c'est ce qui me vient ", tant Marcel Proust est omniprésent dans l'ensemble de son oeuvre, se cache dans la plupart de ses recoins, les plus lumineux comme les plus sombres. Qu'il le prenne comme modèle de ses propres expérimentations dans ses entretiens, puise dans son oeuvre des leçons de composition, partage avec lui le goût des métaphores, déstructure et parodie ses analyses psychologiques, en fasse un personnage de ses romans, le regarde travailler et relire ses épreuves ou décrive avec lyrisme ses phrases "d'une mortelle somptuosité ", il semble le lire et le relire sans fin. En suivant quelques fils de lecture, qui parfois s'entremêlent - la mémoire, les haies d'aubépines, les rats, la peinture ou les poissons cathédrales -, cet article tente de lire Simon lisant Proust, de lire Proust écrit par Simon, de lire Simon en prenant par Proust, de (re)lire Proust à travers Simon, etc. 


\section{Lire Claude Simon lisant Proust Christine Genin Bibliothèque nationale de France}

[C]haque lecteur est, quand il lit, le propre lecteur de soi-même. L'ouvrage de l'écrivain n'est qu'une espèce d'instrument optique qu'il offre au lecteur afin de lui permettre de discerner ce que, sans ce livre il n'eût peut-être pas vu en soi-même ${ }^{1}$.

Il est impossible de ne pas rapprocher les deux grands écrivains du siècle dernier que sont Marcel Proust et Claude Simon, lequel a vu le jour l'année même, 1913, où était publié Du côté de chez Swann. Je n'ai nullement l'ambition de dresser ici un tableau complet de toutes les citations de Proust par Simon, ni de toutes les manières dont leurs deux œuvres sont proches; d'autres s'y sont employé(e)s, dans des monographies, comme Laurence Cadet $^{2}$ et Katerine Gosselin ${ }^{3}$ ou dans la trentaine d'articles disponibles sur le sujet, vers lesquels je

1. Marcel Proust, Le temps retrouvé, dans À la recherche du temps perdu, éd. JeanYves Tadié et coll., Paris, Gallimard, coll. "Bibliothèque de La Pléiade», t. IV, 1989, p. 489-490. Désormais, les références à La recherche du temps perdu renverront toutes à l'édition de la Pléiade préparée par Jean-Yves Tadié et coll. (Paris, Gallimard, coll. «Bibliothèque de la Pléiade», 1987-1989, 4 t.); elles seront indiquées par le sigle $R T P$, suivi du tome en chiffres romains puis de la page, et placées entre parenthèses dans le corps du texte.

2. Laurence Cadet, De Proust à Simon: le miroitement des textes, Paris, Honoré Champion, 2011.

3. Katerine Gosselin, Claude Simon et Marcel Proust: lecture d'une «recherche du temps perdu» simonienne, thèse de doctorat, Université McGill (Montréal), 2010. 
renverrai à l'occasion. Il s'agira seulement pour moi de tirer quelques fils dans l'infinie pelote des correspondances entre les deux œuvres, des fils choisis, de manière nécessairement subjective, parmi les raisons qui font que je trouve mon plaisir de lectrice chez l'un et chez l'autre, chez l'un lisant l'autre.

Le lecteur d'aujourd'hui peut d'ailleurs lire Simon avant Proust. J'ai moi-même beaucoup lu Claude Simon avant de lire en entier et avec attention toute la Recherche. En écrivant le présent article, comme à chaque relecture, $\mathrm{j}$ 'ai encore fait de belles découvertes de lecture chez l'un et l'autre. Le lecteur d'aujourd'hui a lu aussi les études critiques, à propos de l'un, de l'autre ou de leurs ressemblances: «Nous n'avons pas à notre disposition le centième du génie de Proust, mais nous avons sur lui cet avantage (qui est un peu celui de l'âne vivant sur le lion mort) de le lire à partir de ce précisément qu'il a contribué à faire naître - cette littérature moderne qui lui doit tant — et donc de percevoir clairement dans son œuvre ce qui n'y était qu'à l'état naissant ${ }^{4}$ », écrit très justement Gérard Genette. Et, alors qu'il s'apprête à parler de «Flaubert par Proust», il suggère une (ou plutôt des) piste(s) à explorer: "un Flaubert par Proust que nous devons prendre dans les deux sens possibles de cette formule: Flaubert $l u$ par Proust, Flaubert écrit par Proust (sans compter un troisième, peut-être le plus important: Flaubert lu par nous, à travers Proust, en passant ou en prenant par Proust, comme on va à Guermantes en passant par Méséglise $[\ldots])^{5}$ ». Je tenterai donc ici de suivre ces fils de lecture qui s'entremêlent pour notre plus grand plaisir un peu pervers ${ }^{6}$ : lire Simon lisant Proust, lire Proust écrit par Simon, lire Simon en prenant par Proust, (re)lire Proust à travers Simon, etc.

\section{«Proust a ouvert tant de voies»}

Marcel Proust est certainement l'auteur auquel Claude Simon se réfère le plus souvent dans ses entretiens et ses textes théoriques. Katerine Gosselin ${ }^{7}$ dénombre, pour une centaine d'entrevues

4. Gérard Genette, Figures III, Paris, Seuil, 1972, p. 270.

5. Gérard Genette, Palimpsestes. La littérature au second degré, Paris, Seuil, coll. «Essais», 1982, p. 112.

6. «Perversité de l'écrivain (son plaisir d'écrire est sans fonction), double et triple perversité du critique et de son lecteur, à l'infini.» (Roland Barthes, Le plaisir du texte [1973], dans Euvres complètes, Paris, Seuil, 2002, t. IV, p. 228.)

7. Katerine Gosselin, Claude Simon et Marcel Proust, thèse citée, p. 18. 
dépouillées, plus de deux cents apparitions du nom de Proust ou de références à son œuvre.

Simon, qui plus est, porte très tôt un vif intérêt à la Recherche et affirme lire et aimer Proust alors même qu'il est encore peu apprécié par la critique. Dès 1957, il affirme: «Proust a ouvert tant de voies devant nous! L'ingratitude de certains à son égard aujourd'hui me choque un peu $^{8}$...»Et, en 1961: «Mais à dix-huit ans j'avais déjà plongé dans Proust. Quel immense génie ${ }^{9}$ !» «Proust n’est pas une simple référence, il est ce modèle qui guide ses débuts littéraires, ce précurseur qui lui ouvre le champ immense de la littérature moderne ${ }^{10}$ ", écrit Laurence Cadet. Dans les années 1970, Proust sera mis en avant comme précurseur de la littérature moderne, mais ce n'est pas encore le cas en 1945. Jusque dans les années 1950, «Proust n'était pas encore sorti du relatif purgatoire qu'il avait rejoint dans les années 1930. La Recherche était le livre d'une secte; on se le transmettait entre initiés. Le snobisme et l'homosexualité l'isolaient dans un ghetto ${ }^{11} »$, affirme Antoine Compagnon. Il faudra attendre les années 1960 pour voir un renouveau majeur de la critique proustienne, avec Proust et les signes (1964) de Gilles Deleuze, Forme et signification (1962) de Jean Rousset, et, après l'année du centenaire en 1971, Figures III (1972) de Gérard Genette. Les références à Proust deviennent alors de plus en plus fréquentes dans le discours de Simon, qui ressent le besoin de s'en justifier: «Je cite encore Proust, parce qu'il est inépuisable ${ }^{12}$.»

Proust se maintient ensuite comme une référence privilégiée, un modèle lui permettant de confirmer ses propres expérimentations, mais aussi et surtout un immense écrivain qu'il lit et relit et envers lequel il affirme et réaffirme son admiration. En 1981, à Jacqueline Piatier qui lui demande si c'est l'œuvre de Proust qui l'a le plus marqué, il répond: "Je l'ai relue, par morceaux, je ne sais combien de

8. Claude Simon, «Instantané», entretien avec Gabriel d'Aubarède, Les nouvelles littéraires, 7 novembre 1957, p. 7.

9. Claude Simon, «Images de Paris", entretien avec Denise Bourdet, Revue de Paris, janvier 1961, p. 141.

10. Laurence Cadet, De Proust à Simon, ouvr. cité, p. 27.

11. Antoine Compagnon, «Proust et moi», Collège de France [En ligne], consulté le 25 janvier 2015, URL: www.college-de-france.fr/media/antoine-compagnon/ UPL18806_15_A. Compagnon_Proust_et_moi.pdf.

12. Claude Simon, entretiens radiophoniques avec Monique Jonguet, diffusés sur France Culture du 12 au 16 avril 1976, transcrits sous le titre «Entretiens avec Claude Simon ", L'en-je lacanien, vol. 1, n 8, 2007, p. 195. 
fois, et, il y a deux ans, d'un bout à l'autre ${ }^{13}$ »; et à Philippe Sollers, près de vingt ans plus tard, il confirme:

Proust et Céline, ce sont les deux grands écrivains français de la première moitié du $\mathrm{xx}^{\mathrm{e}}$ siècle. [...] Il y a des phrases de Proust qui sont beaucoup plus poétiques que bien des poèmes. [...] Prenez la visite à la marquise de Cambremer, c'est une des choses les plus extraordinaires qu'on ait faites en littérature: cette sensation du temps qui passe, marqué par les changements de couleur des mouettes-nymphéas, c'est prodigieux ${ }^{14}$.

Dans sa correspondance avec Jean Dubuffet, l'écrivain se montre agacé de voir son ami partager la vieille réticence gidienne et lui répond:

Je crois (pardonnez-moi) que s'attacher aux mondanités de Proust n'est pas la bonne façon de le lire. [...] Ce n'est donc pas là que réside pour moi son génie, mais dans l'usage qu'il fait de la description et les structures toutes nouvelles qu'il introduit dans la littérature. En ce sens, je vois en lui l'écrivain le plus subversif (dans tous les sens du terme) du $\mathrm{xx}^{\mathrm{e}}$ siècle ${ }^{15}$.

Il insiste, surtout, dans plusieurs échanges, sur le plaisir que lui procure cette lecture: «Encore une fois, je n'ai pas eu le temps de décortiquer attentivement ce texte. D'autres certainement le feront mieux que moi. Je leur souhaite d'y éprouver encore plus de plaisir que celui que j'ai trouvé moi-même. Proust en est une source inépuisable ${ }^{16} \ldots$.. , ou bien: «éprouvant un grand plaisir à lire une phrase de Proust, j'ai entrepris de la décortiquer, de bien voir comment c'était fait, par quoi mon plaisir avait été suscité, et il en a encore été augmenté $^{17}$...» Et même lorsqu'il prononce une conférence pour analyser son œuvre, il se présente avant tout comme un lecteur passionné:

13. Claude Simon, «Claude Simon ouvre Les géorgiques», entretien avec Jacqueline Piatier, Le Monde, 4 septembre 1981, p. 13.

14. Claude Simon, «La sensation c'est primordial», entretien avec Philippe Sollers, Le Monde des livres, 19 septembre 1997, p. II.

15. Claude Simon, lettre du 21 août 1982, dans Jean Dubuffet et Claude Simon, Correspondance 1970-1984, Paris, L'échoppe, 1994, p. 39-40.

16. Claude Simon, «L'absente de tous bouquets» [1982], dans Quatre conférences, Paris, Minuit, 2012, p. 71.

17. Claude Simon, «Attaques et stimuli», entretien avec Lucien Dällenbach [1987], dans Lucien Dällenbach, Claude Simon, Paris, Seuil, coll. «Les contemporains », 1988, p. 174. 
Je ne suis pas un spécialiste de Proust (seulement un de ses lecteurs passionnés), et je ne prétends pas en apporter une interprétation infaillible. Au surplus, toute interprétation qui se voudrait limitative serait vouée au ridicule, Proust étant fondamentalement, comme tout grand écrivain, polysémique, et son œuvre constituée d'un ensemble subtil de réseaux et de grilles qui se superposent, jouent (dans tous les sens du terme) sur différents registres ${ }^{18}$.

Marcel Proust, on le voit, n'est jamais figé mais plutôt consacré en interlocuteur d'un dialogue que Simon poursuit avec constance tout au long de sa vie, et chez qui il découvre au fil du temps de plus en plus de similitudes avec sa propre conception de l'écriture.

\section{«Le portrait d'une mémoire»}

Pour Proust, l'écriture, on le sait, est aussi une lecture, celle du «livre intérieur de signes inconnus» (RTP, IV, p. 458). En tentant de déchiffrer ce livre et de restituer "l'édifice immense du souvenir» $(R T P, \mathrm{I}, \mathrm{p} .46)$, il décrit de manière très précise des mécanismes de la mémoire et de la conscience que les neurosciences ont depuis explorés ${ }^{19}$. L'importance de la mémoire dans l'écriture de Claude Simon n'est pas non plus à démontrer ${ }^{20}$, et l'expression "portrait d'une mémoire ${ }^{21} »$, qu'il utilise pour décrire son travail dans Le jardin des Plantes, le caractérise probablement beaucoup mieux que le terme d'autobiographie. Le dernier mot de la dernière phrase du dernier roman, Le tramway, qui propose à l'ensemble de l'œuvre un excipit très proustien, est d'ailleurs le mot mémoire: "Comme si quelque chose de plus que l'été n'en finissait pas d'agoniser dans l'étouffante immobilité de l'air où semblait toujours flotter ce voile

18. Claude Simon, «Le poisson cathédrale» [1980], dans Quatre conférences, ouvr. cité, p. 16.

19. Le père et le frère de Proust étaient médecins, et il avait lui-même des connaissances médicales et scientifiques. Voir, à ce propos, Christine Genin, «La culture médicale de Marcel Proust», dans Jean-Yves Tadié et Florence Callu (dir.), Marcel Proust. L'écriture et les arts, Paris, Gallimard, 1999, p. 102-109.

20. Voir, à ce sujet, Christine Genin, L'expérience du lecteur dans les romans de Claude Simon. Lecture studieuse et lecture poignante, Paris, Honoré Champion, 1997 et «Mémoire réticulaire et hypertexte» (sur Claude Simon) [En ligne], janvier 1998, consulté le 25 janvier 2015, URL: http://christinegenin.fr/ blog/2015/05/memoire-reticulaire-et-hypertexte-1998/.

21. «On pourrait dire que le livre est construit comme le portrait d'une mémoire, avec ses circonvolutions, ses associations, ses retours sur elle-même, etc.» (Claude Simon, "Je me suis trouvé dans l'œil du cyclone», entretien avec Antoine de Gaudemar, Libération, 18 septembre 1997, p. III). 
en suspension qu'aucun souffle d'air ne chassait [...] l'impalpable et protecteur brouillard de la mémoire ${ }^{22}$.»

Les mécanismes de la mémoire sont encore loin d'être totalement élucidés, mais chaque souvenir semble être non pas localisé en un point du cerveau, mais réparti dans l'ensemble du réseau cortical, voire dans l'ensemble de l'organisme. La trace mnésique repose sur l'établissement de réseaux plus ou moins durables d'activation préférentielle entre neurones. La mémoire n'est que la réorganisation perpétuellement changeante, au gré des expériences présentes, des sensations passées. Elle est donc tout entière contenue dans le mouvement de son surgissement. Proust ne cesse de mettre en évidence le rôle des émotions dans la remémoration, en évoquant ces instants contingents où un effecteur de mémoire (madeleine, pavés) donne accès à un souvenir. "Chercher? pas seulement: créer» (RTP, I, p. 45), corrige-t-il: la mémoire est une perpétuelle création et tout souvenir une interprétation, au sens musical du terme, de la trace lacunaire des impressions passées.

Dans les romans de Claude Simon, de même, la mémoire est une perpétuelle re-création. Chaque remémoration, que ce soit par la pensée ou a fortiori dans l'écriture, confère au souvenir qu'elle convoque une nouvelle signification. Simon affirme: "On écrit ce qui se passe au présent de l'écriture et ce qui existe dans le souvenir avec toutes les déformations que porte en elle la mémoire et qu'apporte encore l'écriture ${ }^{23} »$. La biographie s'impose à l'écriture, mais s'expose dans le même temps aux transformations que celle-ci est susceptible de lui faire subir: "le souvenir est à la fois antérieur à l'écriture et suscité (ou plutôt enrichi) par elle. Plus on écrit, plus on a de souvenirs ${ }^{24}$.»

Dans ce processus de re-création d'une mémoire par l'écriture, la composition est une question centrale. Simon cite Proust: "Je n'ai qu'un souci qui est la composition" 25 ", lequel ne manque pas de le souligner, par exemple lorsqu'il évoque dans La prisonnière

22. Claude Simon, Le tramway, Paris, Minuit, 2001, p. 141.

23. Claude Simon, «Et à quoi bon inventer?», entretien avec Marianne Alphant, Libération, 31 août 1989, p. 24.

24. Claude Simon, "L'inlassable réa/encrage du vécu», entretien avec Mireille Calle-Gruber, dans Mireille Calle-Gruber (dir.), Claude Simon. Chemins de la mémoire, Grenoble, Presses universitaires de Grenoble, 1993, p. 23.

25. Claude Simon, «Le poisson cathédrale», dans Quatre conférences, ouvr. cité, p. 32 . 
l'unité rétrospectivement découverte par Balzac pour La comédie humaine:

Wagner [...] s'apercevant tout à coup qu'il venait de faire une Tétralogie dut éprouver un peu de la même ivresse que Balzac quand celui-ci, jetant sur ses ouvrages le regard à la fois d'un étranger et d'un père, [...] s'avisa brusquement en projetant sur eux une illumination rétrospective qu'ils seraient plus beaux réunis en un cycle où les mêmes personnages reviendraient et ajouta à son œuvre, en ce raccord, un coup de pinceau, le dernier et le plus sublime. Unité ultérieure, non factice. (RTP, III, p. 666-667)

Unité également toujours précaire: en comparant son œuvre à une robe, Proust fait valoir son droit à l'inachèvement et au rapiéçage - aux collages que ses fameuses paperolles rendent palpables. Simon explique lui aussi le caractère fragmentaire et lacunaire de son écriture en renvoyant, en termes très proustiens ${ }^{26}$, à la nature même de la mémoire, "peuplée» de trous: «c'est un manteau tout déchiré. Proust y faisait allusion dans sa fameuse lettre à Bibesco [...]. Les trous, il faut les laisser - car on les comblerait de façon artificielle par une espèce de ciment de remplissage sans intérêt ${ }^{27} »$. Il importe néanmoins de parvenir par l'écriture à composer avec et à partir de la décomposition, et Simon s'appuie là encore sur l'exemple de Proust: "J'ai, il y a quelques années, à l'occasion d'un colloque sur Proust, entendu avec stupeur (stupeur partagée par Barthes qui était présent et qui a, du coup, renoncé à prendre la parole) un éminent essayiste dire que Proust aurait, comme par une sorte de perversité maligne, "fragmenté le réel" pour déconcerter son lecteur. Or c'est exactement l'inverse ${ }^{28}$ ».

Dans ce travail de composition, les transports analogiques occupent une place primordiale. On sait que l'un des titres de Claude Simon, Les corps conducteurs, est tiré d'une citation de Marcel Proust: "et même une syllabe commune à deux noms différents suffisait à ma mémoire - comme à un électricien qui se

26. Proust écrit dans une lettre de 1913: «on ne rebouche jamais rien dans la vie et les trous vont toujours en s'élargissant", dans Correspondance de Marcel Proust, éd. Philip Kolb, Paris, Plon, t. XII, 1984, p. 191. Voir aussi, Luc Fraisse, «La lentille convexe de Claude Simon», Poétique, nº 117, février 1999, p. 27-46.

27. Claude Simon, «Document. Interview avec Claude Simon», entretien avec Bettina L. Knapp, Kentucky Romance Quarterly, vol. 16, nº 2, 1969, p. 183.

28. Claude Simon, «L’inlassable réa/encrage du vécu», entretien cité, p. 11-12. 
contente du moindre corps conducteur» (RTP, III, p. 358). Simon ne manque jamais d'affirmer que «toute $[s]$ on ouvre est construite sur la nature métaphorique de la langue ${ }^{29}{ }$ et de souligner son intérêt pour «ces transports, au sens étymologique du terme, que sont les métaphores ${ }^{30} »$. Pour Proust comme pour Simon, les métaphores ont à faire avec le fonctionnement analogique de la remémoration, ce que souligne Simon dans son Discours de Stockholm:

Si je ne peux accorder crédit à ce deus ex machina qui fait trop opportunément se rencontrer ou se manquer les personnages d'un récit, en revanche il m'apparaît tout à fait crédible, parce que dans l'ordre sensible des choses, que Proust soit soudain transporté de la cour de l'hôtel des Guermantes sur le parvis de Saint-Marc à Venise par la sensation de deux pavés sous son pied [...] parce qu'entre ces choses, ces réminiscences, ces sensations, existe une évidente communauté de qualités, autrement dit une certaine harmonie, qui, dans ces ensembles, est le fait d'associations, d'assonances, mais peut aussi résulter, comme en peinture ou en musique, de contrastes, d'oppositions ou de dissonances ${ }^{31}$.

Pour Proust, de la même façon, la métaphore n'est pas seulement une figure de style, mais se charge de toute une intensité cognitive: "quand en rapprochant une qualité commune à deux sensations, il dégagera leur essence commune en les réunissant l'une et l'autre pour les soustraire aux contingences du temps, dans une métaphore» (RTP, IV, p. 468).

\section{«Je cite encore Proust»}

À travers ses narrateurs, Simon se met en scène lisant Proust ${ }^{32}$. Il cite aussi très souvent les textes de Proust dans ses propres romans, mais il le fait rarement de manière conventionnelle et convenue:

29. Claude Simon, «Un homme traversé par le travail», entretien avec Alain Poirson et Jean-Paul Goux, La Nouvelle critique, n 105, juin-juillet 1977, p. 42.

30. Claude Simon, "La fiction mot à mot», dans Jean Ricardou et Françoise Van Rossum-Guyon (dir.), Le Nouveau roman: hier, aujourd'hui, actes du colloque tenu à Cerisy en 1971, Paris, Union générale d'éditions, coll. «10/18», 1972, t. II, p. 89-90.

31. Claude Simon, Discours de Stockholm, Paris, Minuit, 1986, p. 22; l'auteur souligne.

32. Par exemple dans La bataille de Pharsale, où il recherche une référence (Paris, Minuit, 1969, p. 20, p. 38, p. 90) et dans Le jardin des Plantes: «Il lit beaucoup (tout Proust $[\ldots]) »($ Paris, Minuit, 1997, p. 332). 
les guillemets sont aussi rares que la mention de la source. Le texte proustien est le plus souvent assimilé, comme absorbé par le texte simonien: "si Simon fait sien le texte proustien, c'est parce que ce texte est sien, comparable ou semblable à soi par essence $[\ldots]$. [...] Simon mêle sa propre voix à la voix proustienne ${ }^{33}$.»

Dès l'ouverture de La corde raide, Simon s'autorise une réécriture de la célébrissime rêverie des chambres qui ouvre la Recherche:

Autrefois je restais tard au lit et j'étais bien. Je fumais des cigarettes, jouissant de mon corps étendu, et je regardais par la fenêtre les branches d'arbres. [...] Au printemps et l'été, à Perpignan, l'acacia multiple se reflétait dans la glace, des fragments verts, le jeu de toutes ses petites feuilles ovales miroitant.

À Paris, dans l'encadrement de la fenêtre, il y avait le flanc d'une maison, un dôme $[\ldots]$ je pouvais voyager et me souvenir des matins où l'on se réveille dans des chambres d'hôtels de villes étrangères ${ }^{34}$.

On connait l'avenir de cet incipit de l'œuvre, qui sera développé dans le motif très important de la fenêtre et sera repris notamment à l'ouverture d'Histoire et à l'excipit de L'acacia.

Le lecteur de Claude Simon remarque aussi très vite que les noms et les traits caractéristiques de certains personnages proustiens sont repris d'un roman à l'autre, attribués par Simon à des personnages différents, qu'ils soient des personnages principaux ou de simples figurants: "Proust et ses personnages ont progressivement investi son monde romanesque au point de s'y fondre et de devenir authentiquement ses propres créatures ${ }^{35} »$. Dès Le tricheur, les

33. Geneviève Dubosclard, «De Marcel Proust à Claude Simon. La mémoire de la création ", dans Sjef Houppermans et coll. (dir.), Marcel Proust aujourd'hui, n ${ }^{\circ} 6$ (Proust dans la littérature contemporaine), 2008, p. 74.

34. Claude Simon, La corde raide, Paris, Le sagittaire, 1947, p. 9. Je citerai ci-dessous les textes de Claude Simon dans leur édition originale: Le tricheur, Paris, Le sagittaire, 1945. Aux éditions de Minuit: L'herbe, 1958; La route des Flandres, 1960; Histoire, 1967; La bataille de Pharsale, 1969; Leçon de choses, 1975; Les géorgiques, 1981; L’acacia, 1989; Le jardin des Plantes, 1997; Le tramway, 2001. Les numéros de pages indiqués ultérieurement le seront tous dans ces éditions, les ouvrages étant signalés par les sigles suivants: CR ( $\mathrm{La}$ corde raide), $\mathrm{T}$ ( $\mathrm{Le}$ tricheur), He (L'herbe), RF (La route des Flandres), Hi (Histoire), BP (La bataille de Pharsale), LC (Leçon de choses), G (Les géorgiques), A (L'acacia), JP (Le jardin des Plantes), Tram (Le tramway).

35. Michel Bertrand, "Personnages proustiens», dans Michel Bertrand (dir.), Dictionnaire Claude Simon, Paris, Honoré Champion, 2013, t. II, p. 811. 
noms d' «Oncle Charles» ( $T$, p. 27, p. 84) et de la "petite Odette» (p. 84) apparaissent; dans L'herbe, la vieille tante à l'agonie porte entre autres le prénom de Léonie ${ }^{36}$; dans Histoire, les allusions au texte proustien se multiplient: le rapport au narrateur et l'histoire amoureuse de l'oncle Charles, père de Corinne, qui s'apparente ellemême à la Gilberte de Proust, rappellent Swann. Les allusions se font parfois beaucoup plus ponctuelles, comme la mention parodique de Saint-Euverte dans Leçon de choses (LC, p. 127).

Les personnages proustiens sont aussi collectifs, comme la petite bande des jeunes filles à Balbec, motif récurrent chez Simon. À la fin de Leçon de choses, Simon évoque par exemple « un groupe de filles assises en rond comme pour quelque cérémonial secret et d'où fusent parfois de frais éclats de rire» (LC, p. 168) sur la plage déserte, évocation qui se clôt par un récital de prénoms féminins appartenant pour certains à la Recherche: «Estelle écoutez moi! Estelle, Élodie, Émilie, Élisabeth, Hélène, Sylvie, Gilberte, Édith, Odette» (LC, p. 172); et le couple dont la rencontre s'entrelace avec la séquence des jeunes filles dans ces pages, trompe un mari qui s'appelle Charles (LC, p. 168). Dans Le tramway, roman à l'atmosphère très proustienne ${ }^{37}$, on retrouve une bande de jeunes filles sur la "plage mondaine»:

Ce qui pour moi faisait tout le prix de ces veillées sur la plage était l'existence dans notre petit groupe d'une fillette [...] qui en avait pris d'autorité le commandement, auréolée à mes yeux [...] du prestige qu'elle tenait de sa fracassante entrée en scène dans une saynète $[. .$.$] montée à l'occasion d'une fête au Cours Maintenon$ où était inscrite ma petite cousine $[\ldots]$ par la suite, je plaquais cette image sur celle du saut à pieds joints d'Andrée, la jeune compagne d'Albertine, par-dessus le "pauv'vieux» assis sur la promenade de Balbec (Tram, p. 51-53).

Michel Bertrand souligne que, dans ce passage souvent commenté, Simon cite délibérément Proust de manière fautive ${ }^{38}$, car ce n'est pas

36. «Marie-Arthémise-Léonie Thomas» (He, p. 47).

37. «Le tramway est ainsi un livre saturé de références proustiennes, qui consonnent exactement avec l'évocation d'un monde disparu, celui de l'ancienne société compartimentée de Perpignan [...] symbolisant toute une époque révolue» (Dominique Rabaté, "Stratégies de la mémoire aujourd'hui», dans Lourdes Carriedo et Luisa Guerrero (dir.), Marcel Proust: écritures, réécritures. Dynamiques de l'échange esthétique, Bruxelles, Peter Lang, 2010, p. 289).

38. Voir Michel Bertrand, «Ironie intertextuelle. Inscriptions proustiennes dans les romans de Claude Simon», dans Joëlle Gardes Tamine, Christine Marcandier 
Andrée qui exécute le saut, mais Gisèle. Cependant, on trouve également dans la Recherche une mention contraire ${ }^{39}$, et cette page du Tramway, comme la liste de Leçon de choses, est justement l'indice du fait que Claude Simon a bien saisi ce qui est l'un des principaux enjeux textuels d'Albertine: son individuation problématique dans la bande des jeunes filles ${ }^{40}$. Cette allusion s'inscrit en outre dans un contexte intertextuel plus large en renvoyant à la manière dont Proust lui-même cite Racine. Non seulement Mme de Maintenon et ses demoiselles sont aussi évoquées dans À l'ombre des jeunes filles en fleurs, à propos des camarades du groom du Grand-Hôtel ${ }^{41}$, mais Andrée se révèle (comme la mère de Proust) férue des tragédies sacrées de Racine, sur lesquelles elle improvise une dissertation si brillante qu'elle met Albertine en sueur ${ }^{42}$.

Comme les personnages et les jeunes filles, les haies d'aubépine(s) chères à Marcel Proust (re)bondissent d'un roman à l'autre. Dans La route des Flandres, elles bordent l'itinéraire des soldats et figurent dans les souvenirs les plus obsédants de Georges: la vision du cheval mort $(R F$, p. 29) et celle du capitaine de Reixach brandissant son sabre avant de se faire tuer $(R F, \mathrm{p} .90$ et à la dernière page, $R F$, p. 314: «le canon se déplaçant insensiblement, le suivant, l'éclat du soleil sur l'acier noir à travers l'odorante et printanière haie d'aubépines»). Les haies d'aubépine(s) reviennent ensuite dans de nombreux romans, le plus souvent dans le contexte de la guerre: "couchés parmi les haies d'aubépine» $(H, \mathrm{p} .101)$, «se tassant contre le pied de la haie sous les petites feuilles d'aubépine» ( $A$, p. 91), dans Histoire et L'acacia; dans Les géorgiques (p. 52, p. 166) et dans Le jardin des Plantes: «Il fera très beau et le soleil printanier brillera sur les prés, les haies d'aubépine en fleurs, les vergers, teintant de saumon

et Vincent Vivès (dir.), Ironies entre dualité et duplicité, Aix-en-Provence, Publications de l'Université de Provence, coll. «Textuelles. Littérature», 2007, p. 225. Proust écrit en outre «c'pauvre vieux» (RTP, II, p. 150).

39. « $[\mathrm{N}]$ ous rencontrâmes presque tout de suite la grande, Andrée, celle qui avait sauté par-dessus le premier président» (RTP, II, p. 237).

40. «[S] on trouble concerne l'individuation même: laquelle des jeunes filles estelle? comment l'extraire et la sélectionner du groupe indivis des jeunes filles?» (Gilles Deleuze, Proust et les signes [1964], Paris, Presses universitaires de France, 1996, p. 214.)

41. RTP, II, p. 66.

42. RTP, II, p. 264-268. Voir Jean-Yves Laurichesse, «Tout au bord du réel. Sur quelques noms propres dans Le tramway», Littératures, $n^{\circ} 46$, printemps 2002, p. $57-75$. 
les fumées qui çà et là s'élèveront parfois de véhicules achevant de se consumer» (JP, p. 160). Dans La bataille de Pharsale, Simon reprend, à plusieurs reprises et de façon plus ou moins tronquée, une citation plus longue: "que nous appelions le raidillon aux aubépines et où vous prétendez que vous êtes tombé dans votre enfance amoureux de moi alors que je vous assure en toute vérité» $(B P$, p. 38, p. 85, p. 92 et p. 181). Comme la mention des jeunes filles dans Le tramway, ces citations obligent en quelque sorte à relire Proust, dont la plupart des lecteurs avaient probablement, comme moi, oublié que le «raidillon aux aubépines» était également, à travers une lettre de Gilberte dans Le temps retrouvé, évoqué comme une scène de guerre et un enjeu de stratégie militaire: "Le petit chemin que vous aimiez tant, que nous appelions le raidillon aux aubépines [...] je ne peux pas vous dire l'importance qu'il a prise. L'immense champ de blé auquel il aboutit, c'est la fameuse cote 307 dont vous avez dî̀ voir le nom revenir si souvent dans les communiqués» (RTP, Iv, p. 335; l'auteur souligne). Passage auquel il convient d'ajouter, pour bien comprendre la citation simonienne, l'aveu précédent de Gilberte concernant la signification du geste qu'elle a eu autrefois dans le raidillon aux aubépines ${ }^{43}$. La guerre militaire vient en quelque sorte redoubler chez Proust comme chez Simon la guerre des sexes. Une autre expression volée chez Proust, «je souffrais comme», revient également très souvent dans La bataille de Pharsale $^{44}$. Là où Proust parle de la guerre et des différences sociales ${ }^{45}$, Simon évoque plutôt la jalousie amoureuse, mais la plainte est commune. Là encore Simon nous invite à relire Proust.

C'est d'autant plus nécessaire que les extraits proustiens cités par Claude Simon sont le plus souvent transformés, découpés, digérés par le texte simonien. Les mutations et les mutilations des textes cités sont en effet nombreuses: prélèvement de fragments très brefs ou réduction du texte d'origine (par la suppression de mots ou

43. «[J]e vous l'ai indiqué d'une façon tellement crue que j'en ai honte maintenant.» (RTP, Iv, p. 269)

44. BP, p. 20 , p. 22 , p. 25 , p. 40 , p. 44 , p. 46, p. 75 , p. 87 , p. 158 et p. 168.

45. "À l'heure du dîner les restaurants étaient pleins et si, passant dans la rue, je voyais un pauvre permissionnaire, échappé pour six jours au risque permanent de la mort, et prêt à repartir pour les tranchées, arrêter un instant ses yeux devant les vitrines illuminées, je souffrais comme à l'hôtel de Balbec quand les pêcheurs nous regardaient dîner, mais je souffrais davantage parce que je savais que la misère du soldat est plus grande que celle du pauvre, les réunissant toutes» (RTP, IV, p. 313). 
l'élagage de vastes tronçons de phrase), vont de pair avec la greffe ou la juxtaposition de divers fragments sans rapport entre eux. Ainsi, dans La bataille de Pharsale, la recherche par le narrateur d'une citation qui lui permettrait d'exprimer sa propre expérience amoureuse — «Disant que la jalousie est comme... comme... Me rappelant l'endroit: environ dans le premier tiers en haut d'une page de droite» (BP, p. 20) - débouche sur une sorte de cut-up, un assemblage syncopé de citations très courtes:

jalousie page de droite vers le haut environ le premier tiers coiffées de ces hauts cylindres édicules Rambuteau s'appelaient des pistières Sans doute dans son enfance n'avait-il pas entendu l'o et cela lui était que nous appelions le raidillon aux aubépines et où vous prétendez que vous êtes tombé dans votre enfance amoureux de moi alors que je vous assure (BP, p. 38; voir aussi p. 90).

Simon reproduit ici non seulement l'expérience physique de la lecture fragmentaire de qui feuillette la Recherche en quête d'une citation précise, mais aussi une manière assez commune de lire Proust par fragments ${ }^{46}$. Ce procédé lui permet enfin de déstructurer l'hypotexte proustien afin d'en faire ressortir certaines assonances dans le signifiant; il permet également de le réécrire autrement: «le texte s'insinue au travers des mots de Proust comme un fil habile, bientôt capable de faire naître un tissu original ${ }^{47}$.»

Il n'hésite pas, par ailleurs, à transformer totalement, voire à caricaturer le texte de Proust; plus bas, une jeune femme dans un train lit un roman de gare évoquant «le tragicaca de conscienza déchirant le héros brûlant d'âmâmour pour la femmenfant du brutal ingénieur kibat les pauvnèg [...] est-ce là pécher ma bite dans son gentil trouducu kefer kefer [...] solution (il sefra finalman mouhane) page 347 et dernière» (BP, p. 178). Et Simon enchaîne avec:

Sodome et Gonorrhée page combien tous laids souvenir voluptueu kil emporté de chézelle lui permetté de sefer unidé dé zatitudezardante zoupâmé kel pouvé tavoir avek d'otr desortekil enarivé taregrété chak plésir kil gougoutait oh près d'aile chak cacaresse invantéé dontil orétu limprudance de lui sinialé ladousseur chak grasse kil lui

46. «(Bonheur de Proust: d'une lecture à l'autre, on ne saute jamais les mêmes passages.)» (Roland Barthes, Le plaisir du texte, dans Euvres complètes, ouvr. cité, p. 224).

47. Laurence Cadet, De Proust à Simon, ouvr. cité, p. 159. 
découvriré kar ilsavé kun instantapré ailezalé tenrichir dinstrument nouvo sonsu plisse (BP, p. 178-179; l'auteur souligne).

Le passage ici cité - qui n'est d'ailleurs pas tiré de Sodome et Gomorrhe mais d'un célèbre passage de $D u$ côté de chez Swann (RTP, I, p. 276) - massacre totalement le texte proustien, le met en pièces et en déforme les signifiants jusqu'à la dénaturation complète de l'énoncé: transcription phonétique, délire orthographique, agglutinations, calembours, troncation fautive, ablation d'une lettre, redoublements syllabiques. Non seulement Simon se livre à une réécriture burlesque de ce passage, mais il raille l'analyse psychologique de Proust en l'assimilant aux lieux communs du roman sentimental de gare traité de la même manière.

Laurence Cadet y voit la nécessité pour l'écrivain d'afficher des «stratégies de destruction et d'absorption ${ }^{48}$ " dans une période (les années 1970) où le Nouveau roman revendique une rupture radicale avec le passé de la littérature: «Proust, l'idole d'hier, semble brûler sur l'autel de sa production romanesque ${ }^{49}$.» Michel Bertrand va plus loin en voyant un meurtre du père littéraire dans cette pratique de $l^{\prime} \ll$ ironie textuelle»:

Claude Simon a trouvé en Marcel Proust un père de substitution. Et ce père, conformément au schéma freudien, Claude Simon l'a conjointement haï et adoré, l'a mis à mort et a assuré sa survie ${ }^{50}$. Si Claude Simon érige Marcel Proust en père de substitution, il ne faut pas se méprendre, il élit l'écrivain, non l'homme. Lecteur de Proust, il lui faut écrire Proust s'il désire devenir écrivain. Il l'écrit donc et l'écrivant il le détruit et le célèbre dans le même mouvement ${ }^{51}$.

Ces lectures sont sans doute exactes, mais j'aime à voir aussi et surtout dans cet usage très libre de la citation le signe d'une avidité dans la dévoration des textes et de la nécessaire irrévérence des artistes entre eux pour être capables de faire une œuvre nouvelle. Simon a souvent affirmé que les citations intertextuelles n'ont pas pour lui un statut différent des autres collages: il colle des extraits de l'œuvre admirée de Proust dans ses textes, et les fait travailler autour d'eux,

48. Laurence Cadet, De Proust à Simon, ouvr. cité, p. 140.

49. Laurence Cadet, De Proust à Simon, ouvr. cité, p. 142.

50. Michel Bertrand, «Ironie intertextuelle», art. cité, p. 218.

51. Michel Bertrand, «Ironie intertextuelle», art. cité, p. 226. 
comme Robert Rauschenberg (qu'il admire et évoque dans Les corps conducteurs notamment) colle des reproductions de tableaux célèbres d'Hokusaï, Piero della Francesca ou Degas parmi d'autres objets dans ses combines paintings. Pour le lecteur, qui remarque l'hommage à Proust et la connaissance très précise de ses textes dont fait preuve Simon, s'amuse de l'ironie, voire de l'irrévérence dont il fait preuve, s'émerveille de la manière dont une citation s'étoile et engendre le texte, va relire Proust en prenant par Claude Simon, l'effet est comparable à celui de ces assemblages artistiques.

\section{L'écrivain comme personnage}

Claude Simon semble en outre faire fi de la démonstration du Contre Sainte-Beuve et n'opère aucune distinction entre l'homme et l'œuvre: il ne se contente pas de citer l'œuvre de Proust mais il en fait un de ses personnages. Dans La bataille de Pharsale, déjà, on pouvait lire:

me représentant toujours Charlus sous les traits de Proust luimême tel qu'il apparaît sur cette photo où l'ombre de la ride qui descend à partir de la narine semble prolonger la moustache comme si celle-ci se relevait en croc ce qui lui confère raidi dans cette pose d'échassier avec sa canne d'ébène son costume sombre ses souliers vernis son col raide sa tête rejetée en arrière $[\ldots]$ une allure vaguement inquiétante clandestine (BP, p. 158).

Dans Le jardin des Plantes, Simon va plus loin en mêlant au sein d'une même évocation sulfureuse l'implicite du romanesque et l'explicite du biographique:

Vers la fin de sa vie, Marcel Proust se faisait apporter dans le bordel pour hommes où il avait ses habitudes des rats qu'il s'amusait à tuer en les transperçant lentement à travers les barreaux de leur cage à l'aide de ces longues aiguilles à chapeaux comme en portaient les femmes à son époque (imaginer leur éclat métallique gris bleu, leurs têtes faites de ces cabochons de jais à facettes). Proust semblait éprouver un grand plaisir à leurs cris en même temps qu'au spectacle de leurs soubresauts et de leurs agonies (JP, p. 106).

Des soldats en permission descendus du front pour quelques jours et venus là pour se faire un peu d'argent en satisfaisant aux goûts de Charlus (en fait, aux goûts de Proust) échangent leurs impressions en attendant dans un salon du bordel pour hommes tenu par Jupien. [...] Proust en train de transpercer d'épingles à 
chapeaux les rats enfermés dans leur cage, tout nu (son corps mou et blanc de malade) ou simplement déculotté (ses yeux globuleux, sa moustache en crocs, son impeccable raie médiane, ses coques défaites et pendantes) secoué par les assauts de l'«apache» qu'il a choisi: «Vous en avez un gros pétard!» dit amoureusement Jupien à Charlus avec une admiration mêlée de respect. (JP, p. 142)

Simon reprend ici, de façon très crue, des anecdotes controversées mais persistantes, colportées par plusieurs contemporains (Sachs, Jouhandeau, Gide), et reprises sans être véritablement confirmées par les biographes successifs ${ }^{52}$, concernant les activités sexuelles de Proust lors de ses nombreuses visites - qu'il décrivait comme documentaires — à l'hôtel Marigny, tenu par Albert Le Cuziat, le modèle de Jupien. Ces passages, dans le contexte du Jardin des Plantes, qui se veut entre le roman et l'essai, participent de la même ironie que les déstructurations textuelles de La bataille de Pharsale; ils suscitent la curiosité du lecteur qui s'en va explorer les traces biographiques de Marcel Proust, et surtout s'interroge sur les motivations de Claude Simon. Ce qui est certain, c'est qu'à partir de ce passage les motifs des rats et de la cage essaiment dans tout le roman. On les retrouve d'abord de façon assez littérale dans la rivière à Calcutta $(J P$, p. 105) ou dans le tableau de Poussin, «la Peste d'Aschrod» (JP, p. 106-107). Mais ils irriguent également, de manière plus métaphorique, les nombreux passages qui évoquent le sadisme et le fantasme d'humiliation dans l'histoire du siècle dernier: la guerre, les camps, l'enfermement, l'extermination ${ }^{53}$.

Les derniers romans de Simon montrent également une autre face, moins sombre quoique tout aussi nocturne, du personnage de Proust: l'écrivain au travail. Dans Le jardin des Plantes, c'est en citant à plusieurs reprises sa correspondance avec ses éditeurs, Gaston Gallimard et Berthe Lemarié, que Simon évoque le travail acharné, fiévreux et presque obsessionnel de relecture, de ratures et d'ajouts de l'écrivain: «Si je ne l'ai pas fait il n'y a qu'à remplacer le mot malheur par le mot chagrin en laissant la phrase pareille.» (JP, p. 138); «seraitce encore temps d'ajouter 3 lignes à une page et un infinitif à l'autre?

52. Voir George D. Painter, Marcel Proust, Paris, Mercure de France, 1966, t. II, p. 335-336; Ghislain de Diesbach, Proust, Paris, Perrin, 1991, p. 623-634; JeanYves Tadié, Marcel Proust, Paris, Gallimard, 1996, p. 788-792.

53. «La cage aux rats de Proust n'en finit pas de fabriquer de sinistres prisons, réelles ou non. [...] la lecture de Proust rencontre celle du $\mathrm{xx}^{\mathrm{e}}$ siècle en une même étreinte sado-masochiste» (Laurence Cadet, De Proust à Simon, ouvr. cité, p. 305-307). 
J'en serais très heureux» ( $J P$, p. 141); "Maintenant que je vous ai, d'artiste à artiste, donné mes raisons» (JP, p. 144). Dans Le tramway, le romancier feint de dénoncer les incohérences proustiennes:

On s'explique mal pourquoi, torturé par la jalousie, le narrateur (qui, comme Proust le laisse entendre, pourrait s'appeler Marcel) confie pour les après-midi la garde d'Albertine à cette Andrée [...]. À noter du reste que les problèmes de vraisemblance n'embarrassent pas Marcel Proust qui, au besoin, recourt sans vergogne aux procédés les plus éculés (comme les hasards, les coïncidences, le fortuit): ainsi, par exemple, lorsqu'il assiste «involontairement» à la fameuse première rencontre de Jupien et de Charlus, ou encore, lorsque "fatigué» il entre - encore "par hasard» et «pour se reposer un peu» — dans le bordel pour hommes tenu par Jupien et «surprend» les innocents propos qu'échangent entre eux les faux apaches fouetteurs (Tram, p. 58-59).

Au-delà du clin d'œil au lecteur du Jardin des Plantes, le jeu avec le lecteur est ici évident: de litotes en guillemets utilisés comme des pincettes, on perçoit que l'ironie est ici une manière d'approuver sans réserve le fait que Proust ne se soucie pas de réalisme, tout en se moquant par avance du lecteur qui ne le comprendrait pas.

Le rapprochement fait ici par Claude Simon entre Marcel et le narrateur résonne avec une conférence que Roland Barthes a consacrée à la fin de sa vie à Proust, et qu'il avoue en introduction avoir failli intituler «Proust et moi»:

en disposant sur une même ligne Proust et moi-même, je ne signifie nullement que je me compare à ce grand écrivain, mais, d'une manière tout à fait différente, que je m'identifie à lui [...] pas à l'auteur prestigieux d'une œuvre monumentale, mais à l'ouvrier, tantôt tourmenté, tantôt exalté, de toute manière modeste, qui a voulu entreprendre une tâche à laquelle, dès l'origine de son projet, il a conféré un caractère absolu. [...] De plus en plus nous nous prenons à aimer non «Proust» (nom civil d'un auteur fiché dans les Histoires de la littérature), mais «Marcel», être singulier, à la fois enfant et adulte, puer senilis, passionné et sage, proie de manies excentriques et lieu d'une réflexion souveraine sur le monde, l'amour, l'art, le temps, la mort ${ }^{54}$.

54. Roland Barthes, «Longtemps je me suis couché de bonne heure», conférence au Collège de France, 19 octobre 1978, dans Euvres complètes, ouvr. cité, t. v, p. 459-465; l'auteur souligne. 
Claude Simon, comme Roland Barthes, s'identifie très probablement d'abord et avant tout à Marcel l'écrivain, mais sans pour autant gommer ni passer sous silence les faiblesses de l'homme.

De fait, dans Le tramway, c'est un peu comme si Claude Simon devenait à son tour un personnage de Proust, l'écrivain Bergotte. L'une des figures du narrateur y est un homme âgé malade qui, dans l'hôpital parisien où il se trouve, pense à la mort. Depuis son lit d'hôpital, il suit par la fenêtre au crépuscule le spectacle d'un couple sur un balcon:

les scènes successives que je suivais avec avidité (apparitions, tentatives d'installation, premier frisson, bavardage) se déroulant pour moi dans le silence, comme un théatre de marionnettes apparaissant et disparaissant [...] jusqu'à ce que bâtiment et ciel se confondent, tout indistinctement englobé de noir où restait seul suspendu un petit rectangle jaune (Tram, p. 127-128).

Passage qui ne peut pas ne pas convoquer pour le lecteur l'icône du «petit pan de mur jaune» contemplé par Bergotte mourant dans $\mathrm{La}$ prisonnière (RTP, III, p. 692).

\section{«Une mortelle somptuosité»}

L'allusion au petit pan de mur jaune le montre, les deux écrivains partagent également un goût pour l'évocation du travail des peintres. Il n'est pas inutile de faire un détour par ce que Proust écrit à propos du célèbre tableau de Chardin, La raie:

une raie est suspendue, dont la vue mêle au désir de la gourmandise le charme curieux du calme ou des tempêtes de la mer dont elle fut le formidable témoin, faisant passer comme un souvenir du Jardin des Plantes à travers un goût de restaurant. Elle est ouverte et vous pouvez admirer la beauté de son architecture délicate et vaste, teintée de sang rouge, de nerfs bleus et de muscles blancs, comme la nef d'une cathédrale polychrome ${ }^{55}$.

On note la présence du Jardin des Plantes, mais c'est surtout une lettre de Lucien Dällenbach à Rainer Warning qui donne au lecteur de Simon l'envie de relire ce texte. Dällenbach, découvrant avec jubilation cet article, y voit rien moins que l'origine de la Recherche et

55. Marcel Proust, «Chardin et Rembrandt», Contre Sainte-Beuve, Paris, Gallimard, coll. «Bibliothèque de la Pléiade», 1971, p. 375-376. 
souligne son intérêt pour Claude Simon: "La raie, la chatte, à côté des poissons morts exorbités, des huîtres et sans doute des moules... Suis mon regard», puis conclut:

À l'instar des fameux rats que Gide évoque dans son «Journal» [...], il suggère qu'une «nature morte», et peut-être une œuvre d'art, pour Proust, est une nature tuée et martyrisée et, puisque la petite Madeleine, on s'en avise maintenant, était déjà rainurée et donc contaminée par la raie, il confirme que l'impureté, la profanation et le coup de griffe de la rature mortifiantes, manifestes à ce stade ultime, pour se faire auparavant plus discrètes, n'en étaient pas moins originaires ${ }^{56} \ldots$

Claude Simon connaissait probablement aussi bien l'article de Proust que ces analyses de son ami Lucien Dällenbach, qui ont peut-être suscité les passages sur les «fameux rats» cités plus haut. Autre correspondance, c'est le passage de la Recherche dans lequel on retrouve, à propos du poisson servi dans la salle à manger du Grand-Hôtel de Balbec, les termes utilisés dans l'article «Chardin et Rembrandt» (notamment la comparaison "comme une polychrome cathédrale de la mer" ${ }^{\prime \prime} »$ ), qui est repris et commenté par Simon dans sa conférence «Le poisson cathédrale». Dans cette conférence, Simon se livre à une analyse précise et technique, qui souligne le caractère novateur de l'utilisation de la description par Proust, et se termine de façon totalement lyrique:

C'est elle [la description] ici qui, maintenant, «fonctionne», travaille, agit. C'est elle qui va et vient, rassemble ce qui était épars, ordonne ce qui paraissait désordre, règle minutieusement les détails de cette grandiose cérémonie où entrent en scène l'univers tout entier, le passé et le présent, le loin et le près, un groom d'hôtel, un poisson, une fleur, les profondeurs de la mer, une vieille marquise $[\ldots]$ tous et tout jouant leur rôle dans cette sorte de système véritablement cosmique où d'un bout à l'autre se répondent les mots, les thèmes mineurs et majeurs entrelacés au sein du grand thème qui domine toute l'œuvre, sans cesse rappelé d'une façon ou d'une autre, [...] l'implacable écoulement

56. Lucien Dällenbach, "À l'origine de la Recherche ou "la raie du jour"», lettre à Rainer Warning, dans Sophie Bertho (dir.), Proust contemporain, Amsterdam, Rodopi, 1994, p. 59.

57. RTP, II, p. 54-55; cité par Claude Simon, «Le poisson cathédrale», dans Quatre conférences, ouvr. cité, p. 12. 
de ce temps recherché, introuvable, omniprésent, perdu à jamais et ressuscité à chaque page par le seul pouvoir de la langue, du texte aux prodigieuses cadences qui, à la fin du livre, se clôt sur lui comme les volets d'un tabernacle dans une phrase d'une mortelle somptuosité ${ }^{58}$.

Il est clair qu'en parlant d'une description proustienne Claude Simon parle aussi de son propre travail, et il est intéressant de retrouver aujourd'hui le passage ci-dessus à son tour cité et commenté ainsi par un autre écrivain, François Bon, qui ne s'y trompe pas:

La leçon de Claude Simon [...] pourrait bien être un de ces textes qui nous aident véritablement à lire Proust aujourd'hui, y compris en ce qu'il nous le démontre techniquement, mécaniquement. [...] Lisons Claude Simon comme s'il parlait non pas de Proust mais de lui-même: Dignité de grand à grand, de venir humblement se saisir en artisan de la phrase même, une seule, pour tout $\operatorname{dire}^{59}$.

Dans une autre conférence, "L'absente de tous bouquets", Simon se livre également à une analyse minutieuse et nuancée d'un passage de Sodome et Gomorrhe qu'il a souvent décrit comme l'un des plus beaux de la littérature française ${ }^{60}$, la visite à la marquise de Cambremer ${ }^{61}$, "quelques pages de Proust qui constituent un petit tableau (en fait, elles aussi, une prodigieuse machine d'horlogerie de précision $)^{62} »$. Dans le chapitre central ${ }^{63}$ du Jardin des Plantes, Simon insère dix-huit fragments ${ }^{64}$ qui proviennent de cette séquence, un contrepoint entre une conversation affectée et prétentieuse sur l'art et la description de vives impressions sensorielles, qu'il met lui-même en contraste avec des passages sur le récit de la débâcle de l'armée française en mai-juin 1940. Les extraits sont cités

58. Claude Simon, «Le poisson cathédrale», dans Quatre conférences, ouvr. cité, p. 37-38.

59. François Bon, Proust est une fiction, Paris, Seuil, 2013, p. 225-230.

60. Voir note 14.

61. RTP, III, p. 200-222.

62. Claude Simon, «L'absente de tous bouquets», dans Quatre conférences, ouvr. cité, p. 64.

63. «Il semble y avoir dans cette disposition une intention de diffuser une lumière proustienne en amont et en aval» (Marie Miguet-Ollagnier, "Le jardin des Plantes à l'ombre de Marcel Proust », Bulletin d'informations proustiennes, $\mathrm{n}^{\circ} 29$, 1998, p. 130).

64. JP, p. 161-162, p. 164 , p. 166 , p. 170, p. 173 , p. 175 , p. 179 , p. 182 , p. 184 , p. $185-$ 186 , p. 189 , p. 200 , p. 202 , p. 203 , p. 205-206, p. 212 et p. $217-218$. 
respectueusement, entre guillemets, dans leur ordre d'apparition (à l'exception d'un retour en arrière, p. 173) et le passage est situé: "Quoique Proust ne donne pas d'indications précises, on peut déduire du contexte qu'il doit être environ cinq heures de l'aprèsmidi» (JP, p. 161). La séquence est empreinte d'un humour subtil que ne manque pas de commenter Simon ${ }^{65}$, et il y est encore question de peinture, à travers la façon dont le snobisme affecte le jugement de la femme du monde sur Poussin. Mais, comme le démontre la lecture de Simon, le principal intérêt littéraire de cette scène mondaine est dans la manière dont Proust y rend à proprement parler visible le passage du temps à travers la couleur des mouettes sur la mer $^{66}$. Grâce au cut-up que Simon opère dans Le jardin des Plantes, l'épisode alterne et contraste en outre avec le récit de la débâcle de l'armée française en 1940. Simon a si bien lu et admiré le texte de Proust qu'il applique et améliore le procédé de contrepoint afin de faire coexister deux moments très différents: «Comme on a essayé de le faire au cinéma, il faudrait plusieurs écrans sur lesquels on projetterait simultanément des images différentes. C'est impossible en parlant ou en écrivant. Mais on peut quand même essayer» ( $J P$, p. 212), commente le narrateur lors de son entretien avec un journaliste. Et, dans un véritable entretien avec un journaliste à propos de son roman, Simon déclare:

On fait comme on peut. On joue avec des alternances, des oppositions, des assonances, des dissonances, etc. Par exemple, quand j'introduis au milieu d'un récit de guerre des citations de Proust, cette conversation paisible - et quelque peu comique - avec la marquise de Cambremer [...] à la même heure [...] où Rommel a lancé son offensive $[\ldots]$. De plus ce passage apparemment futile est peut-être le plus grand tour de force que l'on ait jamais réussi en littérature: aux différentes nuances des couleurs que prennent les mouettes posées sur l'eau [...] on peut sentir au fur et à mesure

65. «Enfin comment ne pas dire un mot de cet humour, parfois attendri [...], parfois féroce $[\ldots]$, toujours sur ce plan un peu mélancolique, à la fois amusé et navré, et qui établit sans cesse cette étonnante distanciation entre le narrateur et ses personnages - y compris ce Marcel au statut tellement ambigu?» (Claude Simon, "L'absente de tous bouquets», dans Quatre conférences, ouvr. cité, p. 70-71).

66. «[D]es mouettes éparses flottaient comme des corolles blanches» (JP, p. 161; RTP, III, p. 203). «[M] ais maintenant elles étaient roses» (JP, p. 218; RTP, III, p. 222). 
de la lecture le passage du temps. De plus encore, il y a parfois des chevauchements, des sortes d'échos ${ }^{67}$.

Au terme de ce parcours trop rapide, il est évident que l'œuvre et la vie de Marcel Proust nourrissent en profondeur la réflexion et l'écriture de Claude Simon, se cachent dans la plupart des recoins de son œuvre, les plus lumineux comme les plus sombres. Il aurait sans doute pu reprendre les mots de Roland Barthes:

l'œuvre de Proust est, du moins pour moi, l'œuvre de référence, la mathésis générale, le mandala de toute la cosmogonie littéraire - comme l'étaient les Lettres de Mme de Sévigné pour la grandmère du narrateur $[. .$.$] ; cela ne veut pas du tout dire que je sois$ un «spécialiste» de Proust: Proust, c'est ce qui me vient, ce n'est pas ce que j'appelle; ce n'est pas une «autorité»; simplement un souvenir circulaire ${ }^{68}$.

Proust, qui avait voulu se purger de son «vice d'imitation" par le pastiche, était particulièrement sensible au fait que l'écriture individuelle émerge toujours de la lecture d'autres écritures qui tour à tour la nourrissent ou la nient. Claude Simon, dont l'œuvre est à son tour une source d'inspiration pour les écrivains d'aujourd'hui, est lui aussi très conscient de cette fructueuse circulation des œuvres, cette innutrition dont parlaient les classiques: «Je place très haut Jean Dubuffet, mais je ne souscris pas à ses anathèmes contre l'asphyxiante culture. La culture n'est asphyxiante que pour les faibles. Pour les autres, elle est au contraire stimulante. Proust était un homme d'une vaste culture, Picasso aussi, Dubuffet aussi ${ }^{69}$...» Et lorsqu'on (re)lit la Recherche on y trouve comme des allusions à l'œuvre de Claude Simon, comme si Proust en était un «plagiaire par anticipation », selon l'expression chère aux oulipiens reprise par Pierre $\operatorname{Bayard}^{70}$ : «Nous ne cessons d'ajouter à la Recherche (comme Proust le faisait sur ses manuscrits), nous ne cessons de l'écrire. Et sans doute c'est cela la lecture», écrit encore Barthes $^{71}$. C'est également ce que Proust souhaitait, qui écrivait:

67. Claude Simon, "Parvenir peu à peu à écrire difficilement», entretien avec Jean-Claude Lebrun, L'Humanité, 13 mars 1998, p. 18.

68. Roland Barthes, Le plaisir du texte, dans Euvres complètes, ouvr. cité, p. 240-241; l'auteur souligne.

69. Claude Simon, «L'inlassable réa/encrage du vécu », entretien cité, p. 60.

70. Pierre Bayard, Le plagiat par anticipation, Paris, Minuit, coll. «Paradoxe», 2009.

71. Roland Barthes, «La chronique» [1979], dans Euvres complètes, ouvr. cité, t. v, p. 628. 
Et c'est là, en effet, un des grands et merveilleux caractères des beaux livres [...] que pour l'auteur ils pourraient s'appeler "Conclusions» et pour le lecteur «Incitations». Nous sentons très bien que notre sagesse commence où celle de l'auteur finit, et nous voudrions qu'il nous donnât des réponses, quand tout ce qu'il peut faire est de nous donner des désirs ${ }^{72}$.

72. Marcel Proust, «Journées de lecture », Contre Sainte-Beuve, ouvr. cité, p. 176-177. 